\title{
First-Principles Study on Electronic Structure and Magnetic Interactions in Ni-doped CdS
}

\author{
Yanrui Guo*, Huiyu Yan, QingGong Song, \\ WeI Kong AND FAng YANG \\ College of Science, Civil Aviation University of China, Tianjin 300300, China \\ Received: 09.11.2020 \& Accepted: 28.05.2021

\begin{abstract}
By using the first-principles calculation method, the electronic structure and magnetic interactions of $\mathrm{Ni}$-doped wurtzite CdS have been investigated. The results reveal that the ground state of the system is a spin polarized state. The magnetic moment of Ni-doped CdS is $2.0 \mu_{\mathrm{B}}$ per cell, which mainly comes from $\mathrm{Ni}$ and its neighbor $\mathrm{S}$ atoms. Various configurations of Ni-doped CdS show half-metallic characters with a $100 \%$ spin polarization. Ni-doped wurtzite CdS presents a long-range ferromagnetic coupling. These results indicate that Ni-doped wurtzite CdS is a promising spin electronic material.
\end{abstract}

topics: first principles, Ni-doped wurtzite CdS, electronic structure, ferromagnetic coupling

\section{Introduction}

During the past decades, dilute magnetic semiconductors (DMSs) have attracted much attention due to their potential as a new functional material, paving the way for the introduction of spin into semiconductor devices [1,2]. Generally, the primary purpose is searching for DMSs with intrinsic ferromagnetism and the Curie temperature $\left(T_{\mathrm{C}}\right)$ higher than room temperature $(\mathrm{RT})$. For this purpose, DMSs with RT ferromagnetic (FM) property, which consisted of a semiconductor doped by $3 d$ transition metal (TM) or nonmagnetic elements, have been realized experimentally, such as Cr-doped ZnTe and GaN [3, 4], TMs-doped $\mathrm{ZnO}, \mathrm{TiO}_{2}, \mathrm{SnO}_{2}$, $\mathrm{Ti}_{2} \mathrm{NiAl}$ [5-10]. However, the origin of RT ferromagnetism can be from intrinsic magnetic behaviors, precipitation of magnetic clusters or the secondary magnetic phases, which is still under debate [11]. These extrinsic magnetic behaviors are undesirable for practices. In order to understand the origin of ferromagnetism in DMSs, many research groups have studied the influence of doping and vacancy on FM properties by using the first-principles calculation method [10-13].

Cadmium sulfide (CdS) is a famous II-VI semiconductor material due to its excellent photoelectric properties. CdS films have been widely used in sensors, solar cells, nonlinear integrated optical devices and other fields $[14,15]$. As already reported, CdS-based DMSs can be realized by transition metals $\mathrm{Mn}, \mathrm{Fe}$, Co and some nonmagnetic dopants as well [16-19]. However, these studies mainly focus on CdS with a zinc blende structure, while there is a shortage of research related to wurtzite $\mathrm{CdS}$.

Therefore, in this paper, based on the firstprinciples calculation method, the electronic structure and magnetic properties of Ni-doped wurtzite CdS are analyzed, and the origin of its magnetism is also discussed. The calculated results show that Ni-doped wurtzite CdS presents a $100 \%$ spin polarization. This research not only helps us to understand the origin of ferromagnetism of DMSs but also supplements the diluted magnetic semiconductor materials.

\section{Details of calculations}

Geometry optimization and energy calculations were performed using density functional theory (DFT) with a plane wave expansion in the Cambridge Serial Total Energy Package (CASTEP) code [20]. The interaction between the electrons and the ionic core is described by an ultrasoft pseudo-potential [21]. The electron-electron exchange and correlation effects are described by the Perdew-Burke-Ernzerhof (PBE) scheme in the generalized gradient approximation (GGA) with and without Hubbard U $[22,23]$. The valence electrons for $\mathrm{S}, \mathrm{Cd}$, and $\mathrm{Ni}$ are chosen as $3 s^{2} 3 p^{4}, 4 d^{10} 5 s^{2}$ and $3 d^{8} 4 s^{2}$, respectively. The energy cutoff is set to $300 \mathrm{eV}$, and a Monkhorst-Pack grid with parameters of $3 \times 3 \times 3$ was used for the irreducible 
The calculated lattice parameters, total energies of intrinsic and Ni-doped CdS.

\begin{tabular}{l|c|c|c|c|c}
\hline \hline & $a[\AA]$ & $c[\AA]$ & $\beta$ & $E_{\text {tot }[\mathrm{eV}]}$ & $E_{\mathrm{g}}[\mathrm{eV}]$ \\
\hline CdS in Ref. [24, 25] & 4.135 & 6.749 & & & 2.42 \\
intrinsic CdS & 4.227 & 6.878 & 120.000 & -3126.08447 & 1.13 \\
Ni-CdS (non-spin polarized state) & 4.198 & 6.840 & 120.004 & -56340.53833 & \\
Ni-CdS (spin polarized state) & 4.202 & 6.834 & 120.004 & -56340.82591 &
\end{tabular}

Brillouin zone. In the geometry optimization process, the SCF tolerance, the maximum tolerances of the force, stress, and displacement were set to $0.5 \times 10^{-6} \mathrm{eV} /$ atom, $0.01 \mathrm{eV} / \AA, 0.02 \mathrm{GPa}$ and $0.5 \times 10^{-3} \AA$, respectively.

It should be noted that both LDA and GGA schemes tend to underestimate the bandgap and give a strong FM coupling interaction [22, 23]. This is caused by the overestimation of delocalization of wave function. There are many approaches to relieve the dilemma, including hybrid potentials methods and the DFT+U scheme [23]. In this paper, besides GGA calculations, GGA+U calculations are also performed to study the magnetic interactions.

In GGA $+U$ calculations, when considering the localization of $\mathrm{Ni} 3 d, \mathrm{Cd} 4 d$ and $\mathrm{S} 2 p$ electrons, and in order to cater for the bandgap of CdS in experiments [24, 25], the on-site Coulomb repulsion $U=7.2 \mathrm{eV}$ and $U=3.87 \mathrm{eV}$ is applied to $\mathrm{Cd} 4 d$ and $\mathrm{S} 2 p$ state, respectively. For the Ni $3 d$ state, there is no experimental value in Ni-doped wurtzite CdS and there is also no theoretical research. Here, in our GGA $+\mathrm{U}$ calculations, we used the same value $U=7.2 \mathrm{eV}$ to the $\mathrm{Ni} 3 d$ state and Cd $4 d$ state, as it was done in other studies [26, 27].

$\mathrm{CdS}$ can present a hexagonal wurtzite structure (space group $P 63 m c$ ) with lattice constants $a=4.14 \AA, c=6.71 \AA$, as reported by [24]. Based on a conventional wurtzite $\mathrm{CdS}$ cell, we build a $3 \times 3 \times 2$ CdS supercell (72 atoms) as a matrix for the doped system, which contains $36 \mathrm{Cd}$ atoms and $36 \mathrm{~S}$ atoms, as shown in Fig. 1.

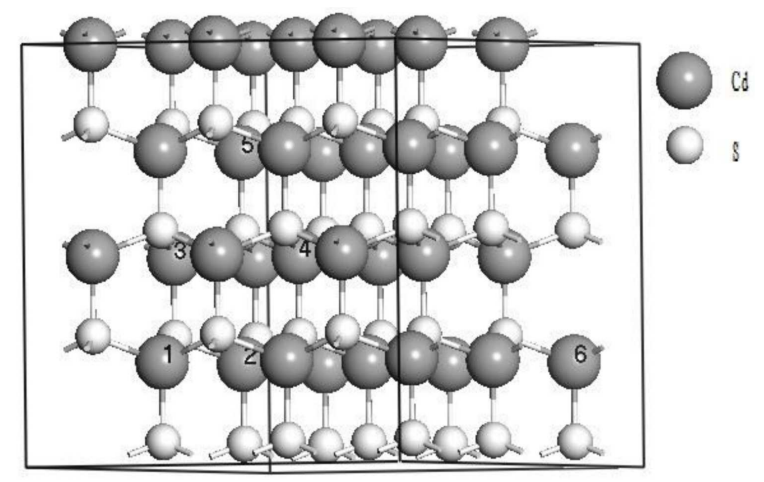

Fig. 1. The supercell structure of CdS.

\section{Results and discussion}

\subsection{Geometry optimization}

Firstly, the geometry optimization of CdS is performed, and the results show that the lattice constants $a$ and $c$ are $4.227 \AA$ and $6.878 \AA$, respectively. These results are in good agreement with the experimental values $(a=4.135 \AA, c=6.749 \AA)[24,25]$ which means that our calculation model and calculation settings are reasonable. The Ni-doped CdS model is established in such a way that a $\mathrm{Ni}$ atom replaces a $\mathrm{Cd}$ atom in a 72 atom supercell. With geometry optimizations, we can get the energies of doped systems with a spin polarized state and a non-spin polarized state, respectively, as shown in Table I. One can see there that the total energy of the $\mathrm{Cd}_{35} \mathrm{NiS}_{36}$ system with the spin polarized state is lower than that with the non-spin polarized state. Therefore, the ground state of Ni-doped CdS is spin polarized. It can also be seen from Table I that the lattice parameters slightly decrease after $\mathrm{Ni}$ doping, which can be related to the fact that the ion radius of $\mathrm{Ni}$ is $0.73 \AA$ smaller than that of $\mathrm{Cd}$ $(0.95 \AA)$. Moreover, the calculated bond length of $\mathrm{Ni}-\mathrm{S}$ in the $\mathrm{NiS}_{4}$ tetrahedron is $2.32 \AA$, i.e., $0.27 \AA$ smaller than for $\mathrm{Cd}-\mathrm{S}(2.59 \AA)$ in the $\mathrm{CdS}_{4}$ tetrahedron. This can also contribute to the difference of lattice parameters.

To test whether CdS can be doped by $\mathrm{Ni}$ atoms, the formation energy of the doped system is calculated using [28, 29]

$$
E_{\text {form }}=E_{\mathrm{Ni}-\mathrm{CdS}}-E_{\mathrm{CdS}}-\mu_{\mathrm{Ni}}+\mu_{\mathrm{Cd}} \text {. }
$$

Here, $E_{\mathrm{Ni}-\mathrm{CdS}}$ is the total energy of the doped system, $E_{\mathrm{CdS}}$ is the total energy of intrinsic $\mathrm{CdS}, \mu_{\mathrm{Ni}}$ and $\mu_{\mathrm{Cd}}$ are the chemical potentials of $\mathrm{Ni}$ and $\mathrm{Cd}$ atom, respectively. The $\mu_{N i}$ is equal to the energy of one $\mathrm{Ni}$ atom in $\mathrm{Ni}$ bulk. Under cadmium-rich conditions, the chemical potential of cadmium is approximately equal to the energy of a $\mathrm{Cd}$ atom in the bulk of metal $\mathrm{Cd}$. Under sulfur-rich conditions, the chemical potential of $\mathrm{S}$ is equal to the energy of an S atom in S bulk. Hence, the chemical potential of $\mathrm{Cd}$ can be obtained by the relation

$$
\mu_{\mathrm{Cd}}=E_{\mathrm{CdS}}-\mu_{\mathrm{S}},
$$

where $\mu_{\mathrm{S}}$ is the chemical potential of S. The formation energy of Ni-doped CdS has been calculated under high cadmium and sulfur conditions, respectively. The results show that the formation energy 

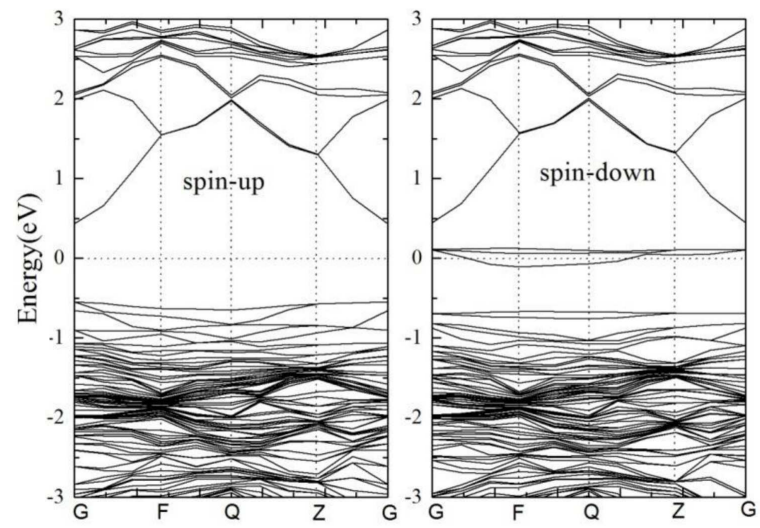

Fig. 2. Band structures of the spin-up and the spin-down of $\mathrm{Cd}_{35} \mathrm{NiS}_{36}$. The zero energy corresponds to the Fermi energy.

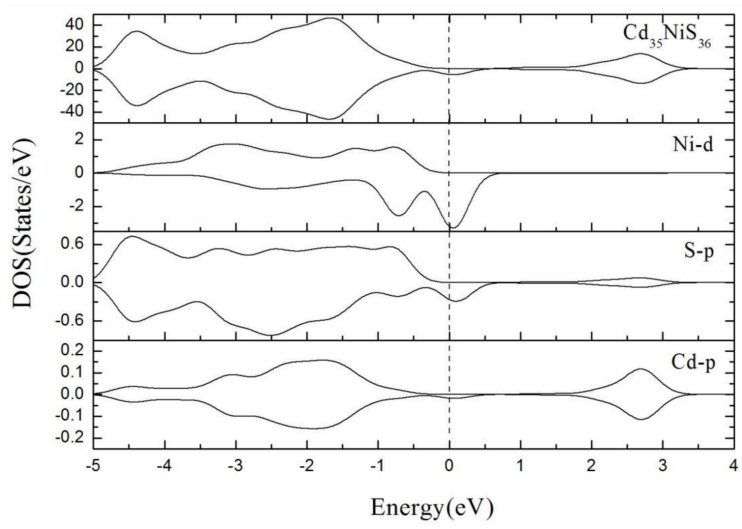

Fig. 3. Total DOS of $\mathrm{Cd}_{35} \mathrm{NiS}_{36}$, partial DOSs of Ni $d, \mathrm{~S} p$ and Cd $p$. The zero energy corresponds to the Fermi energy.

of Ni-doped CdS is (i) $1.95 \mathrm{eV}$ under cadmium-rich conditions, (ii) $0.60 \mathrm{eV}$ under sulfur-rich conditions, which turn out to be far less than the formation energy of the C-doped CdS system (1.2 eV) [16], and also less than the formation energy of the $\mathrm{Cu}$-doped CdS system $(0.73 \mathrm{eV})$ [29]. One can conclude that in the case of rich sulfur, the structure where $\mathrm{Ni}$ replaces $\mathrm{Cd}$ is more stable and can be easily fabricated in experiments.

\subsection{Electronic properties of Ni-doped CdS}

The band structure of Ni-doped CdS is shown in Fig. 2, from which it can be seen that the $\mathrm{Ni}$ dopant can introduce a new impurity energy level into the spin-down band gap. Note that the impurity energy level only appears in the spin-down band, indicating that the doping system presents the half-metallic characteristics with a $100 \%$ spin polarization.

In order to clarify the contribution of different atoms in the Ni-doped CdS system to the spin state, the total and partial density of states (DOSs) of $\mathrm{Cd}_{35} \mathrm{NiS}_{36}$ are analyzed (see Fig. 3 ). There are obvious overlaps among the $\mathrm{Ni} d, \mathrm{~S} p$ and $\mathrm{Cd} p$ states

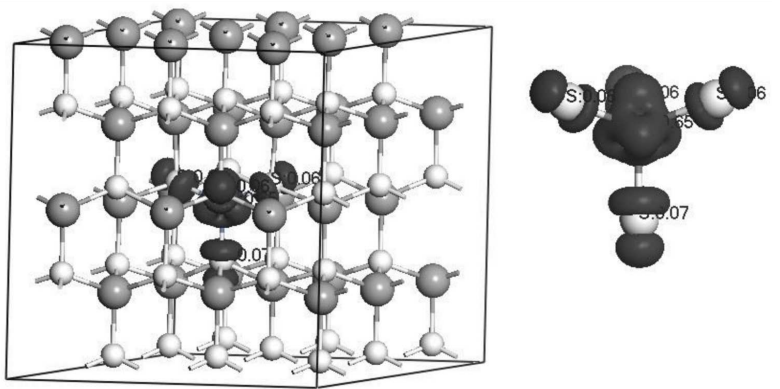

Fig. 4. Spin density distribution of $\mathrm{Ni}$ and its surrounding.

near the Fermi level, indicating that there is a strong $p-d$ hybridization between them. These strong interactions can respond to the splitting of energy levels near the Fermi level. Ni-doped CdS has a total magnetic moment of $2.0 \mu_{\mathrm{B}}$, in which most of the magnetic moments are localized in the $\mathrm{NiS}_{4}$ tetrahedron. The spin density distribution is shown in Fig. 4. We found that the magnetic moment localized on $\mathrm{Ni}$ atom is $0.65 \mu_{\mathrm{B}}$, and the remaining part comes mainly from the nearest sulfur atom around the Ni dopant. The magnetic moment provided by $\mathrm{S}$ at the vertex of the $\mathrm{NiS}_{4}$ tetrahedron is $0.07 \mu_{\mathrm{B}}$, where the magnetic moment contributed by each $\mathrm{S}$ atom is $0.06 \mu_{\mathrm{B}}$.

\subsection{Magnetic coupling of two Ni-doped CdS systems}

To test the ferromagnetic properties of Ni-doped $\mathrm{CdS}$, the magnetic coupling characteristics of $\mathrm{Ni}$ doped system have been studied. We replaced two $\mathrm{Cd}$ by two $\mathrm{Ni}$ atoms, considering different configurations in the $3 \times 3 \times 2 \mathrm{CdS}$ supercell. These configurations are $(1,2),(1,3),(1,4),(1,5)$, and $(1,6)$, where the numbers $1-6$ indicate the positions of the replaced Cd atoms (see Fig. 1). With full geometry optimization of each configuration, the distance between $\mathrm{Ni}-\mathrm{Ni}$ pairs in the five configurations is 4.10, $3.99,5.99,7.98$, and $7.32 \AA$, respectively. The energy calculations of the FM and antiferromagnetic (AFM) states of various configurations were performed and the results are shown in Table II, where $d_{\mathrm{Ni}-\mathrm{Ni}}$ represents the distance between $\mathrm{Ni}$ and $\mathrm{Ni}$, and $M$ is the total magnetic moment of the double doping system. We use $\Delta E=E_{\mathrm{AFM}}-E_{\mathrm{FM}}$ as the criterion of ferromagnetic stability. If $\Delta E$ is positive, the ferromagnetic state of the structure is more stable; otherwise, the antiferromagnetic state is the prior state.

Our results show that $\Delta E$ is greater than zero for all configurations, therefore proving that the ground state of Ni-doped wurtzite CdS prefers a ferromagnetic state. The magnetic moment of various configurations is kept at $4.0 \mu_{\mathrm{B}}$. This means that the half-metal property of $\mathrm{Ni}$-doped $\mathrm{CdS}$ presents robustness - a feature important for the application of spin electronics. We may also note in Table II 
TABLE II

Summary of total energies of FM and AFM states, magnetic moments and energy differences $\left(\Delta E=E_{\mathrm{AFM}}-E_{\mathrm{FM}}\right)$ for different configurations of $\mathrm{Cd}_{34} \mathrm{Ni}_{2} \mathrm{~S}_{36}$ with GGA and GGA+U .

\begin{tabular}{|c|c|c|c|c|c|c|}
\hline \multirow{2}{*}{ Configuration } & \multirow{2}{*}{$d_{\mathrm{Ni}-\mathrm{Ni}}[\AA]$} & \multicolumn{4}{|c|}{ GGA } & \multirow{2}{*}{$\begin{array}{c}\mathrm{GGA}+U \\
\Delta E[\mathrm{meV}]\end{array}$} \\
\hline & & $M\left[\mu_{\mathrm{B}}\right]$ & $E_{\mathrm{FM}}[\mathrm{meV}]$ & $E_{\mathrm{AFM}}[\mathrm{meV}]$ & $\Delta E[\mathrm{meV}]$ & \\
\hline$(1,2)$ & 4.10198 & 4.00 & 22.05 & 140.94 & 118.89 & -5.77 \\
\hline$(1,3)$ & 3.99422 & 4.00 & 0 & 539.51 & 539.51 & 1.11 \\
\hline$(1,4)$ & 5.98655 & 4.00 & 183.43 & 223.64 & 40.22 & -24.82 \\
\hline$(1,5)$ & 7.98203 & 4.00 & 211.68 & 1240.49 & 1028.81 & 53.36 \\
\hline$(1,6)$ & 7.32191 & 4.00 & 197.26 & 812.45 & 615.19 & 12.89 \\
\hline
\end{tabular}

that the ferromagnetic state of the $(1,3)$ configuration has the lowest energy as compared to other configurations so that the total energies are interpreted relative to that of the configuration $(1,3)$ with the FM state. Meanwhile, since the distance between $\mathrm{Ni}$ and $\mathrm{Ni}$ in the $(1,3)$ configuration is the shortest, the two Ni atoms tend to occupy the nearest neighbor position, causing the cluster-like effect.

It is interesting that the $(1,5)$ configuration, in which the distance between $\mathrm{Ni}$ and $\mathrm{Ni}$ is the longest $(7.98 \AA)$, takes the strongest FM coupling with $\Delta E=1028.81 \mathrm{meV}$. A long-range interaction of FM is therefore justified. Due to the periodic properties of models, the magnetic coupling would be then modulated by the neighbor supercell. The $(1,5)$ configuration would be affected by the neighbor unit the most because of the largest distance between $\mathrm{Ni}$ dopants as compared to other calculated configurations. Nevertheless, the FM coupling can still be regarded as a long-range interaction since the distance between the $\mathrm{Ni}$ dopant at site 1 in the neighbor unit and the $\mathrm{Ni}$ dopant at site 5 nearly equals the $\mathrm{Ni}-\mathrm{Ni}$ distance in the $(1,5)$ configuration.

To study the source of ferromagnetism and longrange ferromagnetism of $\mathrm{Ni}$-doped $\mathrm{CdS}$, we mapped the spin resolved band structure of configuration $(1,3)$. This is shown in Fig. 5, from which we can find all the defect levels presenting the main spin states. The $100 \%$ spin polarization rate can be responsible for the integer magnetic moment of the system. The spin density distribution of configuration $(1,3)$ of $\mathrm{Cd}_{34} \mathrm{Ni}_{2} \mathrm{~S}_{36}$ is shown in Fig. 6. There, one can see that spin coupling between the $\mathrm{Ni}-\mathrm{Ni}$ pair can be realized by a bridge connecting the $\mathrm{S}$ atom due to the strong $p-d$ coupling. As a result, $\mathrm{Ni}$ atoms take the same spin orientation, inducing a strong ferromagnetic coupling between them.

To further elucidate the origin of ferromagnetism of Ni-doped CdS, in Fig. 7 we have presented the spin density distribution of configuration $(1,5)$ which shows the maximum $\mathrm{Ni}-\mathrm{Ni}$ distance among all configurations. The ferromagnetic coupling between $\mathrm{Ni}$ atoms at such a large distance cannot be explained by double exchange or super exchange interaction. Our suggestion is that the $\mathrm{Cd}$ atom between two $\mathrm{NiS}_{4}$ tetrahedrons modulates the ferromagnetic coupling between $\mathrm{Ni}$ and $\mathrm{Ni}$. Note that

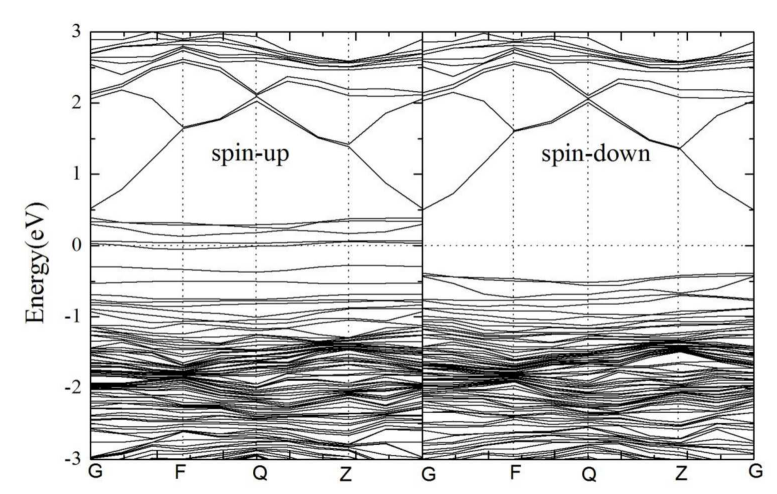

Fig. 5. Band structures of $\mathrm{Ni}$ and its surrounding for configuration $(1,3) \mathrm{Cd}_{34} \mathrm{Ni}_{2} \mathrm{~S}_{36}$. The zero energy corresponds to the Fermi energy.

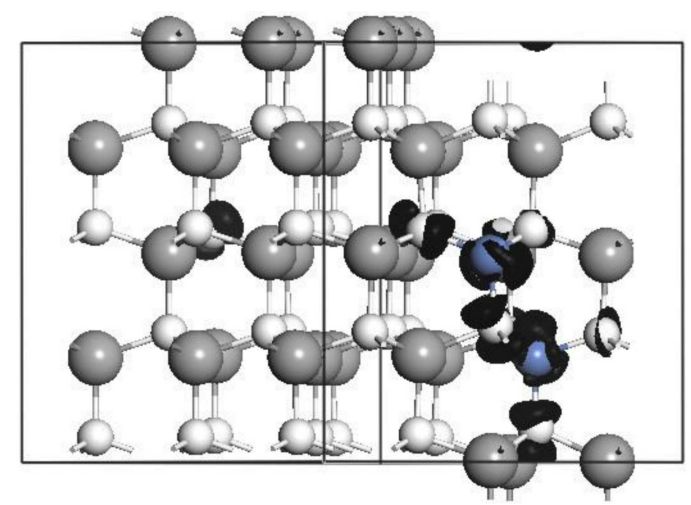

Fig. 6. Spin density distribution of $\mathrm{Ni}$ and its surrounding for configuration $(1,3) \mathrm{Cd}_{34} \mathrm{Ni}_{2} \mathrm{~S}_{36}$.

a strong $p-d$ hybrid between $\mathrm{Ni}$ and the nearest $\mathrm{S}$ atom affects the spin polarization of the $\mathrm{S}$ atom, and such hybridization can modulate the ferromagnetic coupling between $\mathrm{Ni}$ atoms. The second nearest neighbor $\mathrm{Cd}$ atom is coupled with the third nearest $\mathrm{S}$ atom, and the third nearest $\mathrm{S}$ atom is similarly coupled with its nearest $\mathrm{Ni}$ atom, so it gives the $\mathrm{Ni} 3 d-\mathrm{S} 3 p-\mathrm{Cd}-\mathrm{S} 3 p-\mathrm{Ni} 3 d$ interaction chain. This chain of action can be responsible for the longrange coupling between $\mathrm{Ni}$ and $\mathrm{Ni}$.

The modified $\Delta E$ values of various configurations computed using GGA $+U$ are listed in Table II. We found that the Coulomb interaction has a strong 


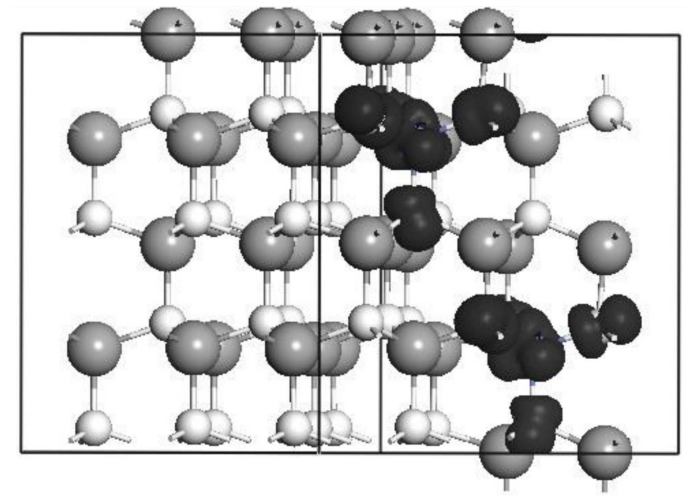

Fig. 7. Spin density distribution of $\mathrm{Ni}$ and its surrounding for configuration $(1,5) \mathrm{Cd}_{34} \mathrm{Ni}_{2} \mathrm{~S}_{36}$.

influence on ferromagnetic coupling in Ni-doped CdS. The magnetic Ni-Ni interaction obtained with the $\mathrm{GGA}+U$ method is much smaller than that obtained with the GGA method. This may originate from the enhancement of localization of corresponding wave functions. As a result, the spatially extended states of electrons decrease, inducing a smaller exchange interaction between $\mathrm{Ni}$ impurities. Similar observations were made in [26]. However, in some other reports, the Hubbard $U$ can enhance magnetic coupling interactions [30]. As the influence of Hubbard $U$ on ferromagnetic stability is absorbing, more detailed research is needed.

The tendency of the coupling strength to vary depending on configurations, shown by the $\mathrm{GGA}+U$ method, is similar to that of the GGA method.

For example, configuration $(1,2)$ and $(1,3)$ present the AFM and the FM ground state, respectively, both, however, take the form of the $\mathrm{Ni}-\mathrm{S}-\mathrm{Ni}$ bridge. This may come from the anisotropy of wurtzite CdS. Configuration $(1,5)$ remains in the FM ground state under the GGA $+U$ calculations, also indicating the long-range interaction of the FM coupling in Ni-doped CdS.

It should be noted that in many DMSs, the spin polarization of $100 \%$ can be realized only in the case of a supercell with one dopant atom. In the case of the supercell with dopant pair configurations, the spin polarization of $100 \%$ is destroyed. In fact, this greatly hinders the application prospect of DMSs. However, in the case of Ni-doped CdS in this work, we have found that all configurations of the supercell with Ni pairs present a $100 \%$ spin polarization. Moreover, as mentioned above, Ni-doped CdS presents a long-range ferromagnetic coupling. These results show that Ni-doped CdS is a promising spin electronic material.

\section{Conclusions}

Based on the first-principles method of density functional theory, the electronic structure and magnetic properties of $\mathrm{Ni}$-doped wurtzite $\mathrm{CdS}$ were studied. The results showed that the ground state of the considered system is a half-metal with a $100 \%$ spin polarization. A nitrogen dopant can introduce a magnetic moment of $2.0 \mu_{\mathrm{B}}$, which comes mainly from the $\mathrm{NiS}_{4}$ tetrahedron. All configurations of Ni-doped wurtzite $\mathrm{CdS}$ allow for keeping a $100 \%$ spin polarization. Also, the long-range ferromagnetic coupling could be realized in Ni-doped wurtzite CdS.

\section{Acknowledgments}

This work was financially supported by the National Natural Science Foundation of China (grant No. 11805272) and the Foundation of the Civil Aviation University of China (grant No. 3122017081).

\section{References}

[1] H. Raebiger, H. Nakayama, T. Fujita, J. Appl. Phys. 115, 012008 (2014).

[2] X.J. Yang, Y.K. Li, C.Y. Shen, B.Q. Si, Y.L. Sun, Q. Tao, G.H. Cao, Z. Xu, F.C. Zhang, Appl. Phys. Lett. 103, 022410 (2013).

[3] H. Saito, V. Zayets, S. Yamagata, K. Ando, Phys. Rev. Lett. 90, 207202 (2003).

[4] R.K. Singh, S.Y. Wu, H.X. Liu, L. Gu, D.J. Smith, N. Newman, Appl. Phys. Lett. 86, 012504 (2005).

[5] L.M.C. Pereira, J.P. Araújo, U. Wahl, S. Decoster, M.J. Van Bael, K. Temst, A. Vantomme, J. Appl. Phys. 113, 023903(2013).

[6] X.P. Wei, Y.D. Chu, X.W. Sun, Y.E.T. Song, P. Guo, Acta Phys. Pol. A 126, 795 (2014).

[7] J.D. Bryan, S.M. Heald, S.A. Chambers, D.R. Gamelin, J. Am. Chem. Soc. 126, 11640 (2004).

[8] K.C. Zhang, Y.F. Li, Y. Liu, F. Chi, J. Appl. Phys. 114, 133707 (2013).

[9] H.X. Luan, C.W. Zhang, F. Li, P. Li, M.J. Ren, M. Yuan, W.X. Ji, P.J. Wang, RSC Adv. 4, 9602 (2014).

[10] M.E. Victor, L.P. William, G.H. Rafael, G.G. Alvaro, Physica B 557, 74 (2019).

[11] T. Dietl, J. Appl. Phys. 103, $07 \mathrm{D} 111$ (2008).

[12] Y.F. Chen, W.B. Mi, J.F. Yang, Q.G. Song, H.Y. Yan, T. Wei, Y.R. Guo, Solid State Commun. 205, 9 (2015).

[13] Q.G. Feng, J. Phys. 32, 445602 (2020).

[14] T. Mandal, C. Dasgupta, P.K. Mait, Phys. Rev. B 91, 104107 (2015).

[15] L. Biadala, H. Frederich, L. Coolen, S. Buil, X. Quélin, C. Javaux, M. Nasilowski, B. Dubertret, J.-P. Hermier, Phys. Rev. B 91, 085416 (2015). 
[16] H. Pan, Y.P. Feng, Q.Y. Wu, Z.G. Huang, J.Y. Lin, Phys. Rev. B 77, 125211 (2008).

[17] H.V. Anh, N.H. Cuong, N. Tu, L.M. Tuan, D.X. Nui, N.D. Dung, N.D.T. Kien, P.T. Huy, D.X. Viet, J. Alloys Compd. 695, 1624 (2017).

[18] C.T. Tsai, S.H. Chen, D.S. Chuu, W.C. Chou, Phys. Rev. B 54, 11555 (1996).

[19] V. Ladizhansky, V. Lyahovitskaya, S. Vega, Phys. Rev. B 60, 8097 (1999).

[20] M.D. Segall, P.J.D. Lindan, M.J. Probert, C.J. Pickard, P.J. Hasnip, S.J. Clark, M.C. Payne, J. Phys. 14, 2717 (2002).

[21] D. Vanderbilt, Phys. Rev. B 41, 7892 (1990).

[22] J.P. Perdew, K. Burke, M. Ernzerhof, Phys. Rev. Lett. 77, 3865 (1996).

[23] V.I. Anisimov, F. Aryasetiawan, A.I. Lichtenstein, J. Phys. Condens. Matter 9, 767 (1997).

[24] A. Nabi, W. Akram, A. Majid, G. Nabi, J. Comput. Intell. Electron. Syst. 2, 116 (2013).
[25] A.I. Oliva, O. Solis-Canto, R. CastroRodriguez, P. Quintana, Thin Solid Films 391, 28 (2001).

[26] W.-Zh. Xiao, L.-L. Wang, L. Xu, Q. Wan, A.L. Pan, H.Q. Deng, Physica B 405, 4858 (2010).

[27] I.S. Elfimov, A. Rusydi, S.I. Csiszar, Z. Hu, H.H. Hsieh, H.-J. Lin, C.T. Chen, R. Liang, G.A. Sawatzky, Phys. Rev. Lett. 98, 137202 (2007).

[28] Q.J. Wang, J.B. Wang, X.L. Zhong, Q.H. Tan, Z. Hu, Y.C. Zhou, Appl. Phys. Lett. 100, 132407 (2012).

[29] P. Li, C.W. Zhang, J. Lian, S. Gao, X. Wang, Solid State Commun. 151, 1712 (2011).

[30] L. Lin, R. Chen, C.Z. He, H.L. Tao, J.T. Huang, L.H. Zhu, L.B. Yan, J.S. Zhang, Vacuum 182, 109681 (2020). 